\title{
UN ACERCAMIENTO EMPÍRICO AL FINANCIAMIENTO DEL DESARROLLO: EL CASO MEXICANO
}

\author{
José Ángel Alcántara Lizárraga* \\ José Luis de la Cruz Gallegos**
}

(Recibido: Junio 2012 / Aprobado: Octubre 2012)

\section{Resumen}

En el presente análisis se estima la relación que para el caso mexicano guarda el crédito otorgado por la banca de desarrollo y el crecimiento económico. Para establecer lo anterior se considera el monto total de crédito destinado por la banca de fomento a los diferentes sectores de la economía. Con el fin de analizar relaciones causales entre las variables se desarrollan vectores autorregresivos y de corrección de error. Las pruebas muestran que no existen relaciones de causalidad entre el crédito y el crecimiento económico. Lo anterior quiere decir que el sistema financiero no influye sobre la productividad de los factores y el crecimiento de México, cuestión que limita el papel que debería desempeñar la banca de desarrollo como complemento del mercado de capitales en la racionalización óptima del crédito.

Palabras clave: cointegración, causalidad, banca de desarrollo, productividad, crecimiento económico

Clasificación JEL: C32, G21, O47

* Investigador del Centro de Investigación en Economía y Negocios, Instituto Tecnológico y de Estudios Superiores de Monterrey, Campus Estado de México. Correo: <maquina_1065@hotmail.com>.

** Director del Centro de Investigación en Economía y Negocios, Instituto Tecnológico y de Estudios Superiores de Monterrey, Campus Estado de México. Correo: <jldg@itesm.mx>. 
José Á. Alcántara / José L. De la Cruz

\section{Abstract}

The present analysis estimates the relation between the development bank and the economic growth. In order to establish such link, the estimations consider the total amount of credit granted by the development bank to the three different sectors of the economy. In order to analyze causal relations among the variables, the study develops autoregressive and error correction vectors. The results show no causality between the development bank and economic growth which implies a set of restrictions in the process that relates the financial system and the total factor productivity as well as economic growth. The latter implies that the financial system does not present any causal effect on the total factor productivity and economic growth which limits the role that development bank has as a complement of the capital markets in the process of rationalization of credit.

Keywords: cointegration, causality, development bank, productivity, economic growth

JEL Classification: C32, G21, O47

\section{Introducción}

El cambio del modelo económico mexicano implementado a principios de la década de los años ochenta significó la transición hacia una nueva forma de entender el papel del Estado en la economía. El carácter proteccionista e intervencionista del gobierno fue sustituido por un espíritu de liberalización del comercio exterior y de la inversión, así como por la adhesión a una política orientada a los mercados (Hanson, 2010).

El colapso de la estrategia de crecimiento hacia adentro enmarcada en el modelo de sustitución de importaciones, obligó al país a reordenar el programa nacional de desarrollo. La base sobre la cual se construyó este nuevo esquema económico se caracterizó por la adhesión a una serie de reformas estructurales encaminadas a conseguir un mayor crecimiento económico y las cuales se vieron reflejadas en la privatización de cerca de mil empresas estatales, la implementación de un nuevo modelo de seguridad social y la liberalización del comercio (Esquivel, 2009).

La unión de México al General Agreement on Tariffs and Trade (GATT) en 1986 y la posterior firma del Tratado de Libre Comercio de América del 
Norte (TLCAN) sellaron la transformación del país a lo que se considera un claro exponente de los lineamientos del tratado de Washington. Existe una extensa literatura acerca de los efectos incipientes que dichas reformas han tenido sobre el crecimiento económico, así como de las causas que han mermado dicho impacto. Hanson (2010) apunta que en el periodo 1985-2008 el producto interno bruto (PIB) per cápita creció a una tasa del $1.1 \%$ anual, sólo por encima de Venezuela que lo hizo a una tasa de $0.8 \%$ y muy por debajo de países como Chile cuya tasa de crecimiento durante ese periodo fue de $4.2 \%$. Por su parte Kehoe y Ruhl (2010) mencionan que el funcionamiento de la economía mexicana ha sido por demás modesto en comparación con países como China, Indonesia y Malasia. En general, el consenso es que el desempeño económico del país ha sido decepcionante en función de las expectativas generadas por el cambio del modelo económico.

A pesar de que México ha logrado alcanzar una inflación controlada y disciplina fiscal, así como un aumento del comercio como proporción del PIB, el país no ha logrado disminuir la brecha de desigualdad que existe entre los estados más ricos de las regiones centro y norte del país y los más pobres al sur del territorio. Además, la baja productividad total de los factores (TPF) ha creado incentivos perversos en el mercado laboral para incrementar la informalidad; efecto que ha sido subsidiado con el aumento de programas de seguridad social para los trabajadores no afiliados. En el periodo 19952004, la TPF solamente creció a una tasa promedio de 1.4\% anual (Levy y Walton, 2009).

Las causas principales del modesto impacto de las reformas en la economía son de distinta naturaleza. Por un lado, existe ineficiencia en los mercados derivada del poder de monopolio que gozan grandes empresas, entre ellas algunas de las que fueron privatizadas en la década de los años noventa, lo que tiene una incidencia directa en el nivel de bienestar social (Urzúa, 2009). En segundo lugar, como se ha mencionado, el comportamiento recesivo de la TPF ha ocasionado una reducción en la acumulación de capital humano, lo que merma el crecimiento.

Aunado a lo anterior, y siguiendo a Hanson (2010), México ha entrado en una fuerte competencia con China en el mercado internacional, en donde se ve en desventaja debido a la naturaleza de los productos que exporta (que comparte en muchos sentidos con el país asiático) y su enorme concentración en el mercado estadounidense.

Una tercera causa es el débil funcionamiento de los mercados financieros. Según Hanson (2010) en el periodo 2001-2008 el cociente de crédito doméstico 
y PIB fue de $18 \%$, sólo a la par de Argentina y Venezuela. En un estudio previo, De la Cruz y Alcántara (2011) concluyen que el crédito otorgado por la banca comercial al sector privado tiene un efecto insípido en el crecimiento económico. De hecho, el país se ha caracterizado históricamente por tener un sistema financiero poco profundo.

Las imperfecciones en el mercado de capitales, principal fuente de financiamiento de la banca privada, han restringido la óptima racionalización del crédito en la economía, lo que en última instancia inhibe las funciones naturales del sistema financiero dirigido al desarrollo y el bienestar. En torno a dichas instituciones, la banca de fomento se caracterizó por ser una de las principales armas del Estado antes de la década de los ochenta para impulsar el crecimiento y el desarrollo. Sin embargo, después de la desregulación de los mercados financieros y la apertura económica de la década siguiente, el campo de acción del crédito para el desarrollo se vio mermado.

Además, la banca privada no ha sido capaz de financiar la inversión y por el contrario, el crédito a las actividades más productivas de México es escaso (De la Cruz y Alcántara, 2011). En este sentido, es de vital importancia comprender los canales a través de los cuales el crédito puede ser causa de un mayor crecimiento y también de desarrollo económico.

Bajo este contexto, el presente trabajo trata de determinar si es que existe una relación de causalidad entre el crédito de la banca de desarrollo y el crecimiento económico para el caso de México. Aunado a lo anterior, se busca establecer si dichos vínculos son de largo plazo. Los vectores autorregresivos (VAR) y de corrección de error (VEC) constituyen una metodología adecuada para determinar si existen interrelaciones causales en el sentido de Granger para los datos en estudio.

El artículo se divide de la siguiente forma: enseguida se hace una reseña de la banca de desarrollo en México, posteriormente se analizan los resultados de las pruebas elaboradas y finalmente se elaboran las conclusiones.

\section{Revisión de la literatura}

Las ideas seminales de Joseph Schumpeter (1911) impulsaron el interés por estudiar el impacto que tiene el desarrollo del sistema financiero sobre el crecimiento económico. En este sentido, a pesar de la extensa literatura en torno a dicha relación, la concepción acerca del papel que desempeña el dinero en una economía ha sido fuente de debate entre los académicos. 
Los economistas más ortodoxos (clásicos) consideraban que el dinero es neutral, por lo que las variables reales (no monetarias) son las únicas capaces de generar un aumento del producto (Miranda, 2009). Por su parte, los economistas keynesianos le dan una fuerte presencia al sistema financiero a través de la banca. Estos pensadores consideran que el dinero no es neutral y puede influir directamente en el funcionamiento de la economía.

En este contexto, Lucas (1988), en un estudio sobre los mecanismos del desarrollo, plantea tres modelos dentro de la perspectiva neoclásica que tratan de explicar la fuente del crecimiento. La primera de ellas tiene que ver con la acumulación del capital físico y el cambio tecnológico; la segunda y tercera consideran la acumulación de capital humano como elemento clave del desarrollo que deriva dicho impacto de la escolaridad y del aprendizaje por la práctica (learning-by-doing). Las ideas de Lucas tienen el común denominador en el canal a través del cual se logran acumular los factores de crecimiento: el sistema financiero. En general, los proyectos de inversión que tienen que ver con la acumulación de capital físico y humano son de largo plazo y los que implican mayor riesgo.

En este sentido, la banca de fomento, quien es la responsable de financiar esta clase de proyectos, ocupa un lugar importante dentro de las implicaciones del modelo.

Durante mucho tiempo las ideas clásicas se vieron plasmadas en los modelos teóricos de crecimiento. La profundidad financiera era considerada una variable exógena en el modelo, lo que mermaba la capacidad que ésta tenía para explicar su impacto en la economía (De la Cruz y Alcántara, 2011). Con el fin de esclarecer el poder explicativo del financiamiento, Pagano (1993) desarrolla un modelo del tipo AK en el cual la tasa de crecimiento de la economía se ve determinada por el sistema financiero a través de tres canales: actuando sobre la tasa de ahorro, la canalización de dicha variable a proyectos de inversión y en el aumento de la productividad marginal social de la misma.

Por otra parte, el modelo de Tirado (2000) muestra de forma precisa al crecimiento económico como una función del sistema financiero, a través de la acumulación de capital humano y los depósitos del sector bancario.

Otros estudios teóricos, como los de Bencivenga y Smith (1991) apuntan que la imposición de restricciones a los mercados de crédito conlleva a consecuencias negativas para la economía. Restringir las tasas de interés, así como aumentar el encaje legal en el sistema bancario tiene un efecto recesivo en el crecimiento económico. 
José Á. Alcántara / José L. De la Cruz

En este esquema, Stiglitz (1998) establece que utilizar al sistema financiero como medio para sustraer rentas del sector privado merma el funcionamiento de la economía debido a que éste sería incapaz de cumplir con sus funciones de aglomeración y distribución del capital a los receptores más productivos.

Al mismo tiempo, Greenwood y Jovanovic (1990) afirman que la intermediación financiera promueve el crecimiento dado que permite obtener una mayor tasa de rendimiento del capital a la vez que el crecimiento "actual" provee los medios de financiamiento para estructuras financieras costosas. Lo anterior describe de forma clara la función natural de la banca de desarrollo como motor de crecimiento económico.

En lo que se refiere al análisis empírico, los estudios seminales de Goldsmith (1969), Shaw (1979) y McKinnon (1979) muestran una relación causal positiva entre el desarrollo del sistema financiero y el crecimiento económico. La hipótesis es corroborada por los trabajos de King y Levine (1993), quienes encontraron resultados de causalidad entre las variables en estudio para una muestra de sección cruzada de 80 países.

Dentro de este contexto, y tratando de dar una justificación a la brecha de desigualdad de la riqueza, Kuznets (1955) plantea la posibilidad de que ésta se incremente durante un periodo para países que pasan de un estado de muy bajo crecimiento a uno mayor. Para el caso de México, la desigualdad del ingreso se ha incrementado desde que el modelo económico actual fue implementado.

\section{La banca de fomento en México}

Con el propósito de comprender mejor el estado de la banca de desarrollo en México con base en un contexto más amplio, resulta adecuada una descripción histórica de su compleja evolución y de sus principales transformaciones. Resulta pues natural dividir el periodo de análisis en dos vertientes claras: una antes de la Revolución Mexicana y otra que sucede dicho acontecimiento.

En lo que se refiere al primer periodo se debe decir que después de la fundación del Nacional Monte de Piedad en 1774, durante el lapso de tiempo que comprendió los años 1821 a 1897 las instituciones de crédito no representaron un papel importante dentro de la economía nacional. Sólo dos bancos figuraron durante dicho periodo: el Banco de Avío, creado en 1830 bajo la tutela de Lucas Alamán y el Banco Nacional de Amortización de Monedas de Cobre, fundado en 1837. Ambas instituciones, la primera con la función de impulsar el desarrollo industrial del país a partir del sector textil 
y la segunda con el objetivo de liquidar la moneda de cobre en circulación, no sobrevivieron ante la inexistencia de un sistema financiero en el país (Huidobro, 2010).

No fue sino hasta 1884 que se instituyó el código de comercio y, con éste, el primer paso hacia un sistema bancario estructurado que terminó por consolidarse en 1897 con la promulgación de la Ley General de Instituciones de Crédito que dividió el sistema en bancos de emisión, hipotecarios y refaccionarios y que permitió la inversión extranjera de bancos en el país. Dicho sistema fracasó con el inicio de la Revolución Mexicana en 1910 y entró en bancarrota en 1914 (Huidobro, 2010).

A partir de la administración de Venustiano Carranza y posteriormente con el gobierno de Álvaro Obregón revivió el concepto de banca de desarrollo nacido en el siglo xix bajo la visión heterodoxa del Estado intervencionista a través de instituciones gubernamentales enfocadas a fortalecer el debilitado aparato productivo.

El periodo posrevolucionario se caracterizó pues por el papel preponderante que el Estado confirió al mercado interno bajo un modelo de Estado de bienestar enmarcado con la nacionalización del sistema financiero. Debido a que el mercado de capitales era débil y poco desarrollado (Levy, 2001), el sistema de financiamiento descansaba bajo un régimen de banca de desarrollo compuesta por las instituciones de crédito y los fideicomisos de fomento económico, entidades paraestatales de carácter financiero especializadas en sectores económicos específicos (Miranda, 2009).

Los bancos de desarrollo se encargaban de financiar las actividades de mayor riesgo y de más largo plazo y el sistema de financiamiento se caracterizaba por ser regulado y orientado hacia actividades específicas generadoras de valor agregado en la economía que se veían subsidiadas con tasas de interés preferenciales y un fácil acceso al crédito.

Así, las funciones de la banca de desarrollo se condensaron en sus inicios en la administración de políticas crediticias políticamente predeterminadas y proveer de servicios financieros a clientes específicos, es así que la banca de fomento surgió con la misión expresa de promover el desarrollo productivo a través de la asistencia técnica y la intermediación financiera de los recursos del gobierno (Luz, 2009). El Banco Nacional de Obras y Servicios Públicos fue creado en 1933 con el objetivo de financiar las inversiones de urbanización. Nacional Financiera (creada un año más tarde), cuya función era apoyar el crecimiento de ramas económicas tales como la petroquímica, del acero, papel, celulosa, cemento, etc., terminó por impulsar a muchas empresas 
paraestatales como respuesta a la Gran Depresión. Por otro lado, el Banco Nacional de Fomento Exterior creado en 1937, tenía como objetivo principal incidir en el aumento de las exportaciones con la intención de mejorar la balanza de cuenta corriente y por lo tanto, el producto (Anaya, 2007). Otras instituciones de banca de fomento que fueron creadas son el Banco Nacional del Ejército, Fuerza Aérea y Armada, el Banco Nacional del Ahorro y Servicios Financieros y la Financiera Rural, entre otras.

El periodo que abarcó los años desde 1940 hasta 1960 representó una época de progreso y crecimiento de los bancos de fomento. Al finalizar el periodo del desarrollo estabilizador, la inestabilidad macroeconómica, la alta inflación, el bajo nivel de ahorro interno y la elevada especulación financiera condujeron a un estancamiento de la banca de desarrollo debido a una reducción del número de clientes, así como de un decremento en el financiamiento de proyectos de largo plazo.

Derivado de lo anterior, se terminó con el compromiso primario de promover el desarrollo de las pequeñas empresas y la función de la banca de desarrollo se concentró en el financiamiento del sector público, de las empresas paraestatales y grandes grupos privados (Luz, et al.). En este contexto, el Estado se vio obligado a la implementación de las reformas estructurales al sector financiero mencionadas con anterioridad.

A partir de la implementación del modelo económico actual, que en el sector financiero se vio reflejada con la liberalización de las tasas de interés y un modelo de financiamiento basado en el mercado de capitales, la banca de desarrollo quedó en segundo plano y sus funciones pasaron a ser de complementariedad con el sector financiero, de tal forma que garantizara la rentabilidad de la banca privada. La idea subyacente de la liberalización de los mercados financieros apuntaba al hecho de que entre mayor fuera el número de intermediarios, menor sería la necesidad de la intervención de la banca de fomento, ya que el mercado por sí mismo canalizaría los recursos a los receptores óptimos.

En un estudio previo, De la Cruz y Alcántara (2011) mencionan que el crédito, al contrario de lo que se pensaba, se otorga para financiar el consumo y actividades del sector servicios en mucho mayor medida de lo que se hace para el sector productivo.

En este sentido, parece que el papel de la banca de fomento está llamado a volver a tomar el rol que le corresponde dentro de la economía nacional. Sin embargo, el financiamiento que recibe de parte de las instituciones para el desarrollo es muy incipiente. Lo anterior supone una contradicción en 
la práctica con respecto al modelo económico teórico implantado a inicios de la década de los noventa. En la Gráfica 1 se puede apreciar una caída de casi el 70\% del crédito total otorgado por la banca de desarrollo en términos reales durante el periodo 1995-2010. El eje de las ordenadas mide el monto del crédito en millones de pesos.

Dicho comportamiento supone que las reformas implementadas bajo el nuevo modelo económico no han sido capaces de utilizar el sistema financiero como un medio para incrementar el crecimiento.

\section{Gráfica 1}

Crédito banca de desarrollo

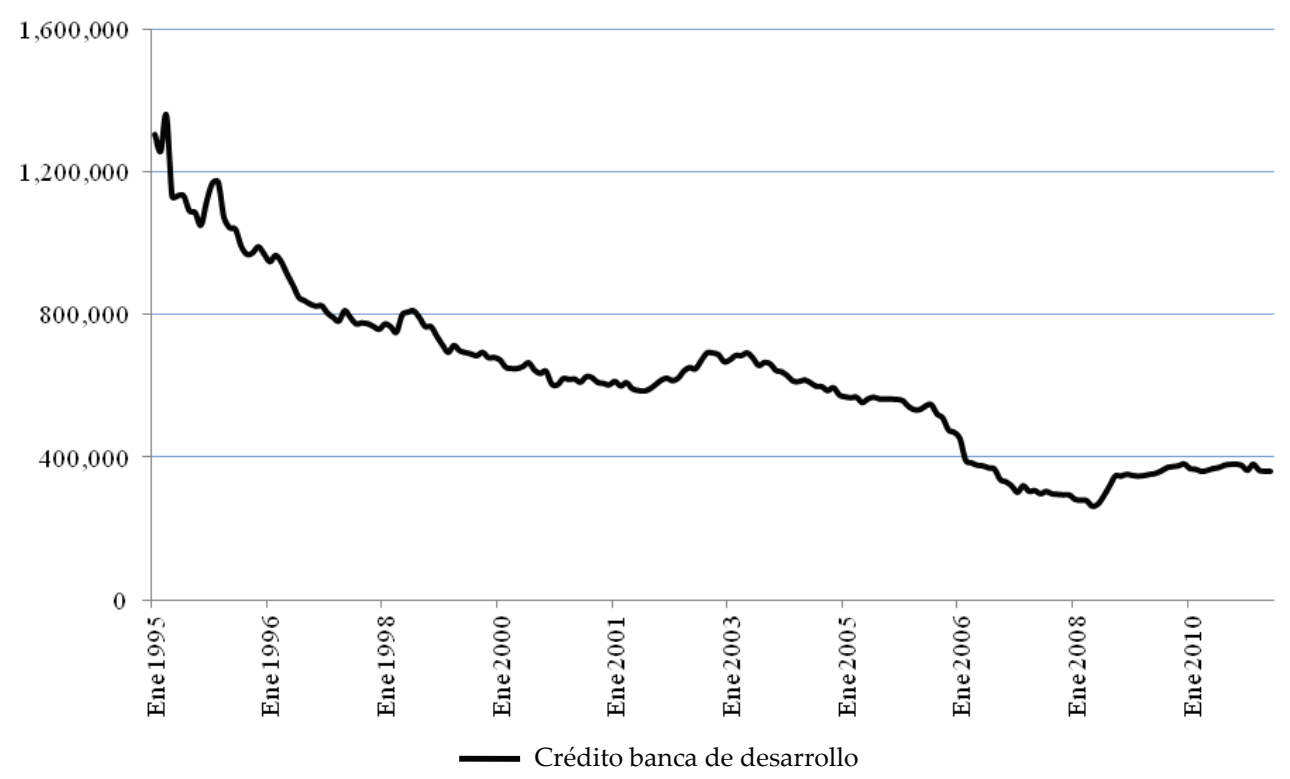

Fuente: elaboración propia.

Por otra parte, en la Gráfica 2 se muestra el crédito otorgado por la banca de fomento a los diferentes sectores de la economía. Se puede apreciar un total abandono al sector primario en comparación con los dos restantes, aunque en términos reales está claro un descenso pronunciado del financiamiento para el desarrollo. 


\section{Gráfica 2}

El crédito sectorial de la banca de desarrollo

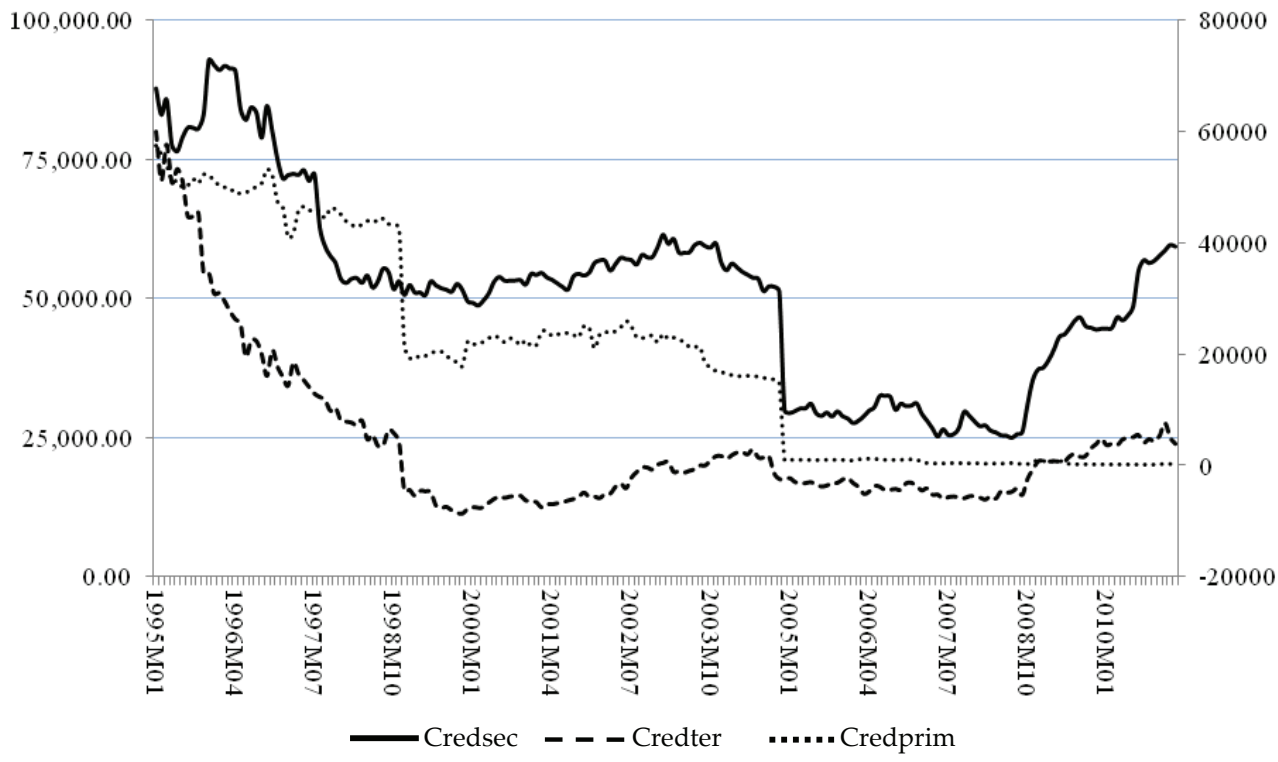

Fuente: elaboración propia.

Como Alcántara y De la Cruz (2011) muestran, el crédito otorgado por la banca comercial al sector privado de la economía no ha sido capaz de racionalizar el financiamiento hacia los sectores que generan el valor agregado y por supuesto, el desarrollo.

El caso del crédito a las actividades primarias ilustra la complejidad del problema que enfrenta actualmente la banca de fomento. Las restricciones a dicho sector contraen naturalmente la frontera de posibilidades de producción del país, lo que en última instancia incrementa la pobreza y disminuye el bienestar (Miranda, 2009).

En lo que corresponde al sector industrial, las empresas, que se enfrentan a un mercado interno inestable, demandan un mayor y más eficiente financiamiento que les permita aumentar su productividad. En contraste, la oferta que el mercado de capitales les presenta es incipiente y obliga al sector privado a subsistir en un entorno de baja competitividad. 
El papel de prestamista de segundo piso de la banca de desarrollo establecido en los lineamientos del esquema de desarrollo no ha funcionado como se esperaba. En el Plan Nacional de Desarrollo (PND) del gobierno de Vicente Fox (2001) se estableció como un instrumento de crecimiento la conformación de un sistema financiero sólido y eficaz en el apoyo del aparato productivo.

El esquema de estrategia tenía dos canales principales: la banca privada y la banca de fomento. En lo referente a la primera, se proponía regresar a los fundamentos de la banca de desarrollo del periodo del desarrollo estabilizador al establecer las condiciones macroeconómicas necesarias para impulsar el financiamiento a las pequeñas y medianas empresas. Según estadísticas de la CANACO (2011), en el mes de mayo de 2011, México ocupó uno de los últimos lugares en el otorgamiento de crédito a las PyMes, que contribuyen el 34.7\% del PIB cuando en 1999 lo hacían en un 42\%.

Por su parte, la banca de desarrolló buscó ser reactivada. Para los inicios de la administración de Fox se proponían en el PND una serie de reformas estructurales y regulatorias de la banca de fomento que tenían que ver con la medición del riesgo y las capacidades administrativas para crear los presupuestos de forma autónoma. Así mismo, se presentó una propuesta para mejorar el acceso de las comunidades rurales al financiamiento.

Para el año 2010, la estructura del crédito otorgado por la banca de desarrollo presentó un comportamiento muy similar al de la banca privada en cuanto al destino del financiamiento se refiere. La Gráfica 3 muestra el comportamiento del crédito otorgado por las instituciones de fomento a la industria manufacturera y aquél destinado a los agentes enfocados al consumo y a los servicios. Resulta evidente la importancia que ha adquirido el crédito que se le otorga al consumo y a los servicios en relación con el que se destina a la industria manufacturera. En términos generales, la banca de fomento no cumple con la función primordial como motor del crecimiento. Lo anterior resalta la complejidad de la situación actual de la banca de fomento en México. Muchos economistas han planteado la importancia de dichas instituciones para las economías emergentes particularmente. De acuerdo con Esquivel (2009), el sistema financiero se presenta como un motor fundamental en la aceleración económica de los países en vías de desarrollo gracias a su capacidad para impactar en lo que el autor denomina las bases del crecimiento de las naciones de América Latina: la igualdad de oportunidades, competencia, estabilidad macroeconómica, y un mayor umbral para la racionalización de los beneficios. 


\section{Gráfica 3}

Crédito banca de desarrollo

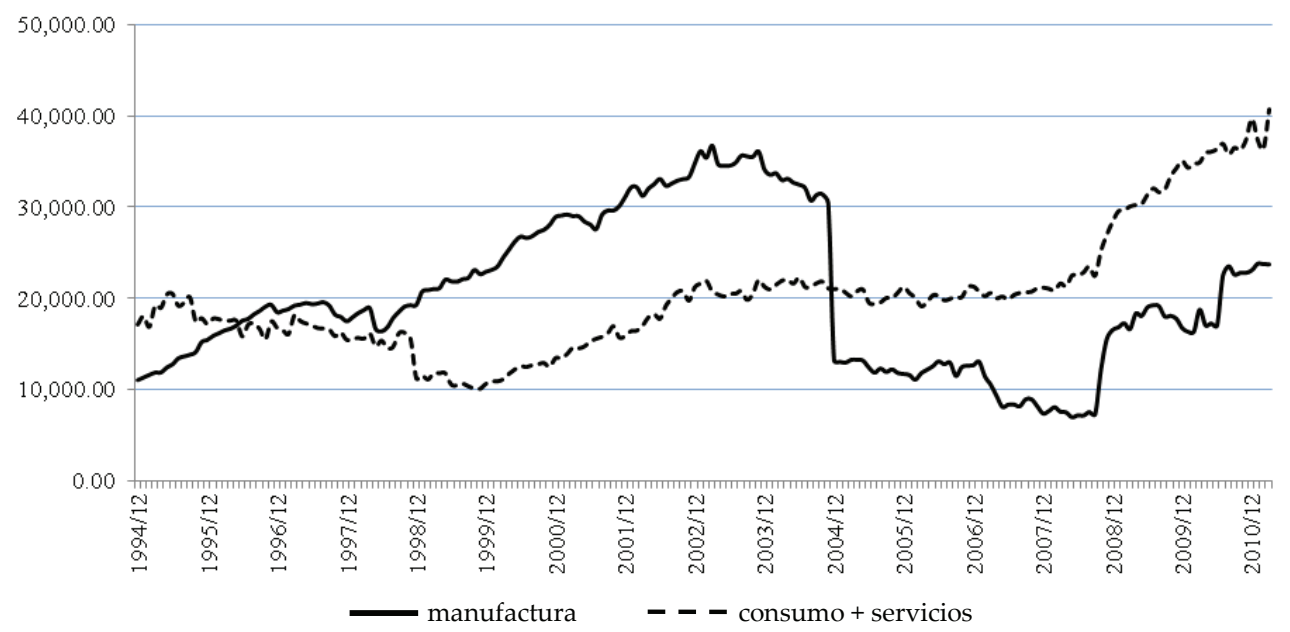

Fuente: elaboración propia.

\section{Metodología y datos}

Para la consecución del objetivo planteado, en primera instancia, se analizan las relaciones causales entre el crecimiento económico y el crédito otorgado por las instituciones de fomento. Los datos utilizados consisten en la desagregación de Índice Global de Actividad Económica (IGAE) ${ }^{1}$ en los diferentes sectores económicos, así como del crédito otorgado por la banca de desarrollo a los mismos. La periodicidad de la información es mensual y el horizonte temporal va de enero de 1995 a noviembre del 2010. Las series correspondientes al crédito de la banca de desarrollo fueron deflactadas con el Índice Nacional de Precios al Consumidor (INPC). Las fuentes de información corresponden a las bases de datos de INEGI y el Banco de México.

En segundo lugar, se busca establecer si hay vínculos de largo plazo entre el crédito otorgado para el desarrollo y la productividad observada Para el cálculo del IGAE se utiliza la misma metodología con la que se calcula el PIB trimestral. Se
considera que este indicador es una buena aproximación del producto interno bruto. 
en México. Para lo anterior se utiliza la serie de productividad de la mano de obra de la industria manufacturera; medida que puede representar de manera adecuada la TFP del país.

El análisis de causalidad parte de la hipótesis nula de que " $Y$ no causa a $X$, dadas otras variables" y se contrasta mediante el estadístico $F$ estándar definido por Ghartey (1993). Siguiendo a Mahía (1999), se realiza el análisis de estacionariedad de las series en estudio con el fin de garantizar el éxito en las etapas de contrastación de hipótesis, por lo que en el caso de que las series no sean estacionarias se hará el análisis de cointegración correspondiente.

Dada la existencia de cambios estructurales en las series económicas mexicanas es de vital importancia determinar de la forma más precisa posible el orden de integración de las mismas, para ello se realizan dos pruebas para la determinación de raíces unitarias. Por un lado se aplica la prueba de Dickey-Fuller Aumentada (ADF) y por otro, la de Kwiatkowski, Phillips, Schmidt y Shin (KPSS).

En este sentido se sigue el proceso de decisión propuesto por Charemza y Deadman (1992) para encontrar el modelo estadísticamente pertinente. Lo descrito es relevante ya que, los trabajos de Toda y Phillips (1993 y 1993) muestran que los modelos autorregresivos no son confiables cuando se estiman relaciones causales en series que no son estacionarias. Algo similar ha sido establecido por Sims, Stock y Watson (1990), quienes señalan que las distribuciones asintóticas no deben ser utilizadas para probar las restricciones en un modelo VAR cuando las variables no son estacionarias. Además, si en un VAR existe un grupo de variables con tendencias estocásticas comunes, se afirma que están cointegradas y entonces puede estimarse un VEC o un VAR, en donde este último debe seguir la propuesta de Toda y Yamamoto (1995). No obstante, Engle y Yoo (1987), Lin y Tsay (1996) y Naka y Tufte (1997) señalan las ventajas de aplicar un VEC. Básicamente los autores muestran que en el largo plazo los resultados del modelo restringido tienen un mejor ajuste. Conforme a estas recomendaciones, en el presente estudio se aplica un VEC para los casos en donde las variables no estacionarias se encuentren cointegradas. La elaboración de un VEC parte de la determinación óptima del número de rezagos a incluir en el modelo, razón por la cual se utilizan los criterios de información. En consecuencia, se construyeron los VAR correspondientes y se determinó el número de rezagos óptimos en función de los criterios de Akaike, Schwarz y Hannan Quinn. Para determinar la posibilidad de que existan relaciones de largo plazo entre las variables se aplicó la prueba de Johansen (1990). En el caso de que las series no estén 
José Á. Alcántara / José L. De la Cruz

cointegradas se elabora un VAR en diferencias para determinar la causalidad sin tener problemas de relaciones espurias.

En términos matemáticos, la elaboración de un VAR se realiza a través de un proceso autorregresivo multivariado del tipo:

$$
y_{t}=\mu+A_{1} y_{t-1}+\ldots+A_{p} y_{t-p}+\varepsilon_{t}
$$

En donde $y_{t}$ es un vector $n x l$ de variables. Si las variables son integradas de orden $1[\mathrm{I}(1)]$ y están cointegradas, el proceso autorregresivo anterior puede ser representado como un VEC de la siguiente manera:

$$
\Delta y_{t}=\mu+\prod y_{t-1}+\sum_{i=1}^{p-1} \Gamma_{i} \Delta y_{t-i}+\varepsilon_{t}
$$

donde

$$
\Pi=\sum_{i=1}^{p} A_{i}-\mathrm{I} \quad \text { y } \Gamma_{i}=-\sum_{j=i+1}^{p} A_{j}
$$

El rango de $\Pi$ determinará el número de vectores de cointegración linealmente independientes que pueden existir entre los componentes de $y_{t}$.

Sea $r$ el rango de la matriz $\Pi$ y $k$ su número de columnas. Si $\Pi$ es de rango reducido, es decir, $r<k$, entonces decimos que existen dos matrices que tampoco son de rango completo, $\alpha$ y $\beta$, tal que $\Pi=\alpha \beta$ y $\beta y_{t-1}$ es I(0), en donde $\beta^{\prime}$ representa los vectores de cointegración y $\alpha$ los parámetros de ajuste del desequilibrio en el proceso. Por lo tanto, el problema se reduce a encontrar $r$. La causalidad se determina aplicando una prueba $F$ sobre los rezagos de las variables bajo estudio.

\section{Resultados}

En primer lugar, se determinó el orden de integración de la series en estudio. Para lo anterior se utilizaron las pruebas ADF y KPSS para la detección de raíces unitarias. Los resultados obtenidos establecen que todas las series son integradas de orden $1[\mathrm{I}(1)] .^{2}$

2 En la Tabla 1a y 1 b del Apéndice 1 se muestran los resultados de ambas pruebas. 
Debido a que las series no son estacionarias, se estudia la posibilidad de que tengan una relación en el largo plazo. Para considerar dicha posibilidad, se utiliza la metodología de Johansen para la determinación de ecuaciones de cointegración. En la Tabla 2 del Apéndice 2 se muestran las relaciones para las cuales se encontraron vínculos de largo plazo.

Las pruebas indican que hay relación de largo plazo entre el crédito que otorga la banca de desarrollo al sector terciario y el IGAE. De igual manera, se encuentra tal relación entre el índice de productividad de la mano de obra manufacturera y el crédito otorgado por la banca de desarrollo a nivel agregado. Para las series restantes no se encontraron resultados estadísticamente significativos para llegar a la conclusión de que se encuentran cointegradas.

Con base en los resultados obtenidos, se aplican las pruebas de causalidad en el sentido de Granger. Sólo para las series de productividad y crédito a nivel agregado se aplicó la prueba sobre un vector de corrección de error. La Tabla 1 muestra los resultados obtenidos para ambas familias de series.

Tabla 1

Causalidad en el sentido de Granger

\begin{tabular}{|c|c|c|c|c|c|}
\hline Variable dependiente & Crédito & Credprim & Credsec & Credter & Causalidad \\
\hline IGAE & 0.6968 & 0.8172 & 0.24 & 0.5331 (VEC) & No hay causalidad \\
\hline \multicolumn{2}{|c|}{ Variable dependiente } & \multicolumn{2}{|l|}{ IGAE } & \multicolumn{2}{|c|}{ Causalidad } \\
\hline \multicolumn{2}{|l|}{ Crédito } & \multicolumn{2}{|l|}{0.3142} & \multicolumn{2}{|c|}{ No hay causalidad } \\
\hline \multicolumn{2}{|l|}{ Credprim } & \multicolumn{2}{|l|}{0.5477} & \multicolumn{2}{|c|}{ No hay causalidad } \\
\hline \multicolumn{2}{|l|}{ Credsec } & \multicolumn{2}{|l|}{0.8322} & \multicolumn{2}{|c|}{ No hay causalidad } \\
\hline \multicolumn{2}{|l|}{ Credter } & \multicolumn{2}{|c|}{0.9215 (VEC) } & \multicolumn{2}{|c|}{ No hay causalidad } \\
\hline \multicolumn{2}{|c|}{ Variable dependiente } & \multicolumn{2}{|c|}{ Crédito } & \multirow{2}{*}{\multicolumn{2}{|c|}{ No hay causalidad }} \\
\hline Productividad & & \multicolumn{2}{|c|}{0.5788 (VEC) } & & \\
\hline
\end{tabular}

Fuente: elaboración propia. 
José Á. Alcántara / José L. De la Cruz

No se encuentran resultados estadísticamente significativos de causalidad entre el crédito otorgado por la banca de fomento y el crecimiento económico. Los resultados contradicen de manera puntual la función nominal de las instituciones de fomento en México. Dichos organismos no son capaces de generar mayor producción $y$, por consecuencia, están muy lejos de tener un efecto causal en el desarrollo. Los resultados de la serie de productividad respaldan el hecho de que es prácticamente nulo el efecto del crédito hacia los niveles de vida de la población.

A este respecto, se ha encontrado que la productividad de la mano de obra del sector manufacturero tiene una relación de largo plazo con el crédito otorgado por la banca de desarrollo, sin embargo no fueron encontrados resultados significativos de causalidad entre ambas. La implicación económica fundamental de lo anterior radica en el hecho de que las reformas potenciales que vayan orientadas a fortalecer el modelo neoliberal se verán mermadas en su capacidad de acción debido a las restricciones del sistema financiero, que no es capaz de redistribuir el capital hacia los sectores productivos del país.

Los resultados afirman la idea de que la función de la banca de desarrollo en México está desviada de su concepción original, por lo que sólo existe para financiar el crédito otorgado por la banca privada, que va dirigido en su gran mayoría al consumo privado.

Se ha señalad o que el lento crecimiento de la TFP es un factor determinante para explicar la lentitud con que avanza la economía mexicana. En palabras de Levy (2010), una forma de incentivar dicha variable es a través de la óptima racionalización del financiamiento, que en última instancia colocaría la inversión de capital en sus mejores usos.

En la Tabla 2a del Apéndice 2 se muestran los resultados obtenidos de las pruebas de causalidad para el caso de la productividad, medida por la productividad de la mano de obra en la industria manufacturera (PLM) y el número de trabajadores ubicados en la informalidad. La frecuencia de los datos es trimestral y comprende el periodo 2005-2010. Las pruebas dan evidencia de una relación de causalidad unidireccional negativa que va de la productividad hacia la informalidad, aunque no se encontraron relaciones de largo plazo entre ellas.

Lo anterior implica que una mejora en la productividad total de los factores mejoraría la estructura del mercado laboral mexicano al reducir la informalidad e incentivar el crecimiento económico. No obstante lo anterior, 
en la Tabla 4a del Apéndice 4 se muestra que el crédito de la banca de desarrollo no tiene ninguna relación causal con el empleo. ${ }^{3}$

Aunado a lo anterior, los resultados para el caso del crédito al desarrollo y la productividad no dan evidencia de una relación entre las variables. Lo anterior supone una ruptura en el proceso natural que empieza con el sistema financiero y termina con el aumento en la producción. La evidencia empírica plantea el efecto nulo del crédito de la banca de desarrollo hacia el crecimiento económico.

Ante este resultado, es importante cuestionarse acerca de los casos exitosos de la banca de fomento como institución para financiar el desarrollo. Un ejemplo de esta naturaleza tiene que ver con el sistema financiero alemán. Una economía con instituciones sociales y corporativas muy arraigadas y ligadas históricamente a la ciudadanía así como con mercados financieros relativamente competitivos, tiene un sistema financiero fuerte con la capacidad de prescindir del mercado de capitales. Cabe aclarar que la banca extranjera en Alemania representa sólo el 6.6\% del total del mercado.

Las empresas transnacionales obtienen su financiamiento a través de la banca privada y del mercado de capitales, mientras que las pequeñas y medianas empresas así como las personas físicas, lo hacen a través de la banca de desarrollo.

La función clave de la banca de fomento en dicho país es garantizar el ajuste estructural de la economía alemana (Golonbek, 2008) a través de los siguientes canales: apoyo a las PyMes, crédito a la vivienda, financiamiento a la tecnología y a la innovación y promoción del sector educativo.

Casos como el brasileño y el chileno son muy interesantes ya que son muy similares al mexicano. A pesar de que la banca de desarrollo actúa como una institución de segunda instancia para corregir las fallas del mercado de capitales, tiene objetivos muy claros acerca del destino del crédito y el nivel de riesgo de las inversiones que financia. Lo anterior supone que la implementación de una reforma integral del sistema de la banca de fomento es clave para incentivar el crecimiento económico.

La forma de reactivar la productividad de los sectores potencia está en función de la capacidad que tenga la banca de desarrollo de llegar a dichos sectores de la economía.

3 Para la realización de estas pruebas se analizan las series del crédito total otorgado por la banca de desarrollo a la economía mexicana y los trabajadores permanentes asegurados del IMSS. La frecuencia de las series es mensual y comprenden el periodo 1995-2011. Las series son integradas de orden 1. 
José Á. Alcántara / José L. De la Cruz

\section{Conclusiones}

El modelo económico implementado a finales de la década de los ochenta no ha tenido el impacto estimado sobre la economía nacional. Lo anterior supone una serie de restricciones de los efectos de las reformas estructurales llevadas a cabo a partir de 1994, dentro de las que se encuentran medidas para incrementar la profundidad del sistema financiero, las cuales no han ayudado o a financiar las actividades que generan el valor agregado en la economía.

En este sentido, los resultados plantean tres hechos relevantes. En primer lugar, que el crédito otorgado por la banca de desarrollo no tiene efecto sobre el crecimiento económico. Lo anterior implica una restricción directa de financiamiento para los proyectos de inversión productivos más riesgosos y de más largo plazo. A su vez, el crédito otorgado por la banca comercial al sector privado se encuentra concentrado en el consumo y el sector servicios, lo que tergiversa la función original de la banca de fomento.

En segundo lugar, los resultados demuestran que la productividad de la mano de obra de la industria manufacturera no se encuentra relacionada causalmente con el crédito otorgado por la banca de fomento. Lo anterior interrumpe el canal por el cual el sistema financiero puede transmitir crecimiento a la economía. Al encontrarse relaciones de causalidad bidireccional negativa entre la productividad y la informalidad, y al ser el crédito de la banca de fomento incapaz de incidir en la PLM, el crecimiento económico se ve mermado.

Finalmente, se comprueba que el crédito otorgado por la banca de fomento no tiene impacto sobre el empleo y por lo tanto es incapaz de incidir en el bienestar de la población. A pesar de que dicho tipo de crédito funcionó como una de las principales herramientas del Estado para incidir en el crecimiento, ahora representa sólo un subsidio a un crédito perecedero.

Se plantea que la banca de desarrollo debe dejar de actuar como una institución prestamista de segundo piso. Más bien, debe comportarse como una banca independiente de la banca privada y del mercado de capitales con objetivos bien definidos de tal forma que el sistema financiero funcione a través de una economía social de mercado en vez de una de libre mercado. 


\section{Apéndices}

\section{Apéndice 1. Pruebas de raíz unitaria}

Tabla 1a

Análisis de raíz unitaria (ADF)

\begin{tabular}{|lccccc|}
\hline \multicolumn{1}{|c}{ Serie $(\boldsymbol{l n})$} & $\begin{array}{c}\text { Estadístico } t \\
\text { (en niveles) }\end{array}$ & $\begin{array}{c}p \text {-value } \\
(\mathbf{e n ~ n i v e l e s )})\end{array}$ & $\begin{array}{c}\text { Estadístico } t \\
\left(\Delta y_{t}\right)\end{array}$ & $\begin{array}{c}p \text {-value } \\
\left(\Delta y_{t}\right)\end{array}$ & $\mathrm{I}(\boldsymbol{d})$ \\
IGAE & -2.850591 & 0.1814 & -6.252711 & 0.0000 & $\mathrm{I}(1)$ \\
IGAESEC & -2.570579 & 0.1010 & -9.399404 & 0.0000 & $\mathrm{I}(1)$ \\
IGAEPRIM & -2.682543 & 0.2452 & -14.81696 & 0.0000 & $\mathrm{I}(1)$ \\
IGAETER & -1.709968 & 0.7433 & -14.01843 & 0.0000 & $\mathrm{I}(1)$ \\
CREDITO & -3.423774 & 0.0512 & -16.80399 & 0.0000 & $\mathrm{I}(1)$ \\
CREDPRIM & -2.686423 & $0.0073^{*}$ & -12.66722 & 0.0000 & $\mathrm{I}(0)$ \\
CREDSEC & -1.414538 & 0.1461 & -12.90195 & 0.0000 & $\mathrm{I}(1)$ \\
CREDTER & -2.336021 & 0.1619 & -3.615726 & 0.0063 & $\mathrm{I}(1)$ \\
PRODUCTIVIDAD & -2.753447 & 0.2168 & -4.854252 & 0.0005 & $\mathrm{I}(1)$ \\
& & & & & \\
\hline
\end{tabular}

* Se toman los resultados de la prueba de Kwiatkowski-Phillips-Schmidt-Shin (KPSS).

Fuente: elaboración propia.

Tabla $1 b$

Análisis de raíz unitaria (KPSS) ${ }^{4}$

\begin{tabular}{|c|c|c|c|c|c|}
\hline Serie $(\ln )$ & $\begin{array}{l}\text { Estadístico } \\
\text { (en niveles) }\end{array}$ & $\begin{array}{c}\text { Valor crítico } \\
5 \% \\
\text { (en niveles) }\end{array}$ & $\begin{array}{c}\text { Estadístico } \\
\left(\Delta y_{t}\right)\end{array}$ & $\begin{array}{c}\text { Valor } \\
\text { crítico5\% } \\
\left(\Delta y_{t}\right)\end{array}$ & $\mathrm{I}(d)$ \\
\hline IGAE & 0.235760 & 0.146000 & 0.080297 & 0.146000 & $\mathrm{I}(1)$ \\
\hline IGAESEC & 0.249664 & 0.146000 & 0.082674 & 0.146000 & $\mathrm{I}(1)$ \\
\hline IGAEPRIM & 0.166534 & 0.146000 & 0.243324 & 0.463000 & $\mathrm{I}(1)$ \\
\hline IGAETER & 0.139784 & 0.146000 & & & $\mathrm{I}(0)$ \\
\hline CREDITO/DESARROLLO & 0.169425 & 0.146000 & 0.139894 & 0.146000 & $\mathrm{I}(1)$ \\
\hline CREDPRIM & 0.201084 & 0.146000 & 0.034709 & 0.146000 & $\mathrm{I}(1)$ \\
\hline CREDSEC & 0.175193 & 0.146000 & 0.087283 & 0.146000 & $\mathrm{I}(1)$ \\
\hline CREDTER & 0.318467 & 0.146000 & 0.360659 & 0.146000 & $\mathrm{I}(2)$ \\
\hline PRODUCTIVIDAD & 0.162161 & 0.146000 & 0.014572 & 0.146000 & $\mathrm{I}(2)$ \\
\hline INFORMALIDAD & & & & & \\
\hline
\end{tabular}

Fuente: elaboración propia.

4 En el caso de las series del IGAE para el sector terciario y el crédito al consumo, tomamos el resultado de la prueba ADF. 
José Á. Alcántara / José L. De la Cruz

Apéndice 2. El crédito, la productividad y la informalidad

Tabla 2a

Causalidad en el sentido de Granger

\begin{tabular}{|llc|}
\hline Variable dependiente & PRODUCTIVIDAD & Causalidad \\
Informalidad & 0.0278 & informalidad $\rightarrow$ productividad \\
Variable dependiente & INFORMALIDAD & \\
Productividad & 0.0469 & productividad $\rightarrow$ informalidad \\
\hline
\end{tabular}

Fuente: elaboración propia.

Tabla $2 b$

Causalidad en el sentido de Granger

\begin{tabular}{|llc|}
\hline Variable dependiente & CRÉDITO AL DESARROLLO & Causalidad \\
Informalidad & 0.7629 & No hay causalidad \\
Variable dependiente & INFORMALIDAD & \\
Crédito al desarrollo & 0.1049 & No hay causalidad
\end{tabular}

Fuente: elaboración propia. 


\title{
Apéndice 3. Ecuaciones de cointegración
}

\section{Tabla 3a}

Ecuaciones de cointegración

\begin{tabular}{|lcccc|}
\hline Variables & Autovalor & Traza & $\mathbf{5 \%}$ & Núm. de CE(s) \\
& & & & \\
IGAE-CREDTER & 153.5479 & 159.0366 & 18.39771 & NINGUNA $^{* *}$ \\
& 5.488717 & 5.488717 & 3.841466 & 1 \\
CRÉDITO-PRODUCTIVIDAD & & & & \\
& 25.43788 & 25.43788 & 18.39771 & NINGUNA $^{* *}$ \\
& 3.257361 & 3.841466 & 3.851466 & 1 \\
\hline
\end{tabular}

Series: CRÉDITO AL DESARROLLO (agregado y al sector primario), IGAEPRIM, PRODUCTIVIDAD. ** Denota rechazo de la hipótesis nula a un nivel de significancia de 5\%.

Fuente: elaboración propia.

\section{Apéndice 4. Crédito de la banca de desarrollo y el empleo}

\author{
Tabla 4a
}

Causalidad en el sentido de Granger

\begin{tabular}{|lll|}
\hline Variable dependiente & EMPLEO & Causalidad \\
Crédito desarrollo & 0.2717 & No hay causalidad \\
Variable dependiente & CRÉDITO DESARROLLO & \\
Empleo & 0.8070 & No hay causalidad \\
\hline
\end{tabular}

Fuente: elaboración propia. 
José Á. Alcántara / José L. De la Cruz

\section{Referencias bibliográficas}

Anaya, M. (2007), "La banca de desarrollo en México", CEPAL, Serie Financiamiento del Desarrollo, núm. 190, Santiago de Chile.

Arias, J., O. Azuara, P. Bernal, J. Heckman y C. Villareal (2010), “Policies to Promote Growth and Economic Efficiency in Mexico", Institute for the Study of Labor, Bonn, Alemania.

Bencivenga, V. y B. Smith (1991), "Financial Intermediation and Endogenous

Growth", The Review of Economic Studies, vol. 58, núm. 2, 195-209.

Charemza, W. y D.F. Deadman (1992), New Directions in Econometric Practice: General to Specific Modeling, Cointegration, and Vector Autoregression, Aldershot, Edward Elgar Publishing.

De la Cruz, J.L., J.A. Alcántara (2011), “El desarrollo del sistema financiero y el crecimiento económico: un análisis de causalidad para México", Manuscrito.

Engle, R. y B. Yoo (1987), "Forecasting and Testing in Co-integrated Systems", Journal of Econometrics, vol. 35, 143-159.

Esquivel, G. y F. Hernández-Trillo (2009), “How Can Reforms Help Deliver Growth in Mexico?" en L. Rojas-Suárez (ed.) (2009), Growing Pains in Latin America, Washington, DC, Center for Global Development, pp. 192-235.

Ghartey, E. (1993), “Causal Relationship between Exports and Economic Growth: Some Empirical Evidence in Taiwan, Japan and the US", Applied Economics, vol. 25, núm. 12, 1145-52.

Goldsmith, W. (1969), Financial Structure and Development, New Heaven, Yale University Press.

Golonbek, C. y E. Sevilla (2008), “Un estudio de casos sobre banca de desarrollo y agencias de fomento", Centro de economía y finanzas para el desarrollo de la Argentina, Documento de trabajo núm. 20, Argentina.

Greenwood, J. y B. Jovanovic (1990), "Financial Development, Growth and the Distribution of Income", The Journal of Political Economy, vol. 98, núm. 5, 1076-1107.

Hanson, G. (2010), “Why isn't Mexico rich?", Journal of Economic Literature, vol. 48, núm. 4, 987-1004.

Huidobro, A. (2010), “Contribución de la banca de desarrollo mexicana al financiamiento bancario a empresas", Tesis, Universidad Anáhuac del Norte, México.

Kehoe, T. y K. Ruhl (2010), "Why Have Economic Reforms in Mexico Not Generated Growth", Federal Reserve Bank of Minneapolis, EU. 
King, R. y R. Levine (1993), “Finance and Growth: Schumpeter Might be Right”, The Quarterly Journal of Economics, vol. 108, núm. 103, MIT Press, 717-737. Kuznets, S. (1955), "Economic growth and income inequality", The American Economic Review, vol. 45, núm. 1, 1-28.

Levy, E., A. Micco y U. Panizza (2004), "Should the Government Be in the Banking Business? The role of the state owned and development banks", BID, Departamento de Investigación.

Levy, S. y M. Walton (2009), No Growth Without Equity? Inequality, Interests, and Competition in Mexico, The World Bank, Washington.

Lin, J. y R. Tsay (1996), "Cointegration Constrain and Forecasting: An Empirical Examination", Journal of Applied Econometrics, vol. 11, 519-538.

Lucas, R. (1988), "On the Mechanics of Economic Development", Journal of Monetary Economics, vol. 22, Chicago University, 3-42.

Luz, M. (2009), “Nacional Financiera: balances y perspectivas del pilar industrial de la banca de desarrollo", Economía informa, núm. 361, UNAM, México.

Mahía, R. (1999), “Revisión de los procesos de análisis de la estacionariedad de las series temporales", Universidad Autónoma de Madrid, España.

McKinnon, R. (1973), Money and Capital in Economic Development, Washington, DC, Brookings Institution.

Miranda, M. (2009), “La banca de desarrollo en México 1940-2009: ¿instrumento para el desarrollo?", Economía informa, núm. 361. UNAM, México, 68-79.

Naka, A. y D. Tufte (1997), "Examining Impulse Response Functions in Cointegrated Systems", Applied Economics, vol. 29, núm. 12, 1593-1603.

Pagano, M. (1993), "Financial Markets and Growth, an Overview", European Economic Review, vol. 37, nos. 2 y 3, 613-622.

Schumpeter, J. (1911), "The theory of economic development", The European Heritage in Economics and the Social Sciences, Harvard University Press, 61116.

Shaw, E. (1973), "Financial deepening in economic development", Oxford University Press.

Sims, C., J. Stock y M. Watson (1990), "Inference in Linear Time Series Models With Some Unit Roots", Econometrica, vol. 58, 113-144.

Stiglitz, J. (1998), "The Role of Financial System in Development", LAC ABCDE, San Salvador, El Salvador.

Toda, H. y P. Phillips (1993), "Vector Autoregressions and Causality", Econometrica, vol. 61, núm. 6, 1367-1393. 
José Á. Alcántara / José L. De la Cruz

Toda H. y I. Yamamoto (1995), “Statistical Inference in Vector Autoregressions with Possibility Integrated Process, Journal of Econometrics, vol. 66, 225-50. Tirado, J. (2000), “Crecimiento con cambio tecnológico endógeno, bancos y dinero", Estudios económicos, vol.15, núm. 1, UAM.

Urzúa, C. (2009), "Efectos sobre el bienestar social de las empresas con poder de mercado en México", Finanzas públicas, vol. 1, núm. 1, México. 